\title{
Establishment and Application of Heminested RT-PCR Assay for Detection of Mosquito-Borne Flavivirus — Guizhou Province, China, 2018
}

\author{
Zhijing Xue ${ }^{1,2}$; Ning Zhao'; Jun Wang'; Xiuping Song'; Fengxia Meng'; Wenqin Liang ${ }^{1}$; \\ Jingzhu Zhou ${ }^{3}$; Dan Wang'; Zhong Zhang ${ }^{4,5, * ;}$; Qiyong Liu ${ }^{1, *}$
}

\begin{abstract}
Introduction: Mosquito-borne flavivirus can lead to serious infectious diseases worldwide and cause high mortality and disability. In order to strengthen epidemiological investigation of flavivirus and meet the needs of early warning of diseases, a simple, rapid, and sensitive detection method needs to be established to prevent and control mosquito-borne diseases.
\end{abstract}

Methods: Using the NS5 gene of flavivirus in GenBank, 3 universal primers targeting the conserved regions were designed. The complementary DNAs (cDNAs) of Japanese encephalitis virus (JEV), dengue virus (DENV), West Nile virus (WNV), and yellow fever virus (YFV) were used as the template to optimize the reaction conditions. A heminested reversetranscriptase polymerase chain reaction (hnRT-PCR) was established to verify the sensitivity and specificity of this method and to detect field-caught mosquitoes.

Results: Our results showed that this method exhibited better specificity, higher sensitivity, and the ability to detect multiple viruses simultaneously. The lowest detection limit of JEV, DENV-2, YFV, and WNV was $3 \times 10^{4}, 3 \times 10^{6}, 3 \times 10^{5}$, and $3 \times 10^{4}$ copies $/ \mu \mathrm{L}$, respectively. Mosquito-borne flavivirus was successfully detected in the field-caught mosquito samples using the method established in this study.

Discussion: The hnRT-PCR method established in this study can be employed for the rapid detection of flavivirus and provide technical support for early and rapid diagnosis of mosquito-borne flavivirus.

\section{INTRODUCTION}

Mosquito-borne viruses can be transmitted through mosquito bites on sensitive vertebrates and cause natural-focus infection diseases worldwide (1). Flavivirus is one of the most important mosquitoborne viruses, including Japanese encephalitis virus (JEV), dengue virus (DENV), West Nile virus
(WNV), yellow fever virus (YFV), etc., which can lead to fever, headache, hemorrhagic fever, and encephalitis (2). Flaviviruses therefore are responsible for considerable morbidity and mortality and have become a serious public health problem worldwide (3).

It was reported that dengue fever was endemic in more than 100 countries worldwide, threatening the health of more than 2.5 billion people (4). JE had occurred everywhere in China except for Xinjiang, Tibet, and Qinghai, and it was the most harmful mosquito-borne infectious disease with high morbidity and mortality (3). WNV was imported into New York city in 1999 and spread widely in the neighboring states for 5 years (5). In recent years due to global warming, the rapid development of tourism, and continuous changes in the ecological environment, the incidence of mosquito-borne diseases has been on the rise, which brings new challenges to the prevention and control of mosquito-borne diseases (G). Therefore, efficient detection and diagnosis of mosquito-borne viruses is urgently needed to prevent and control mosquito-borne diseases. Currently, conventional flavivirus diagnostic methodologies include virus isolation and culturing methods, serological methods, and nucleic acid detection methods. However, virus culture is relatively laborious and time-consuming, and serological methods lack of specificity and are prone to cross-reactions between viruses (7-8). Compared with traditional virus isolation and serological methods, nucleic acid detection technology has unique advantages in early detection of mosquito-borne viruses.

\section{METHODS}

\section{Primer Design}

The complete genomic sequences of mosquito-borne flavivirus were downloaded from the GenBank 
database and aligned using the Clustal W program (Table 1). A stretch of nucleotides conserved in the strains was identified and the primers were designed using the Primers Express software. The primer sequences and characteristics are shown in Table 2.

\section{Viruses, Viral RNA Extraction, and Reverse Transcription}

Viral strains, including JEV, DENV-2, YFV, WNV, Sindbis virus (SINV), and chikungunya virus (CHIKV), were provided by the National Institute for Viral Disease Control and Prevention of China CDC. Viral RNA was extracted using the Rneasy MiNi Kit (QIAGEN, Germany) and used directly for complementary DNA (cDNA) synthesis using QuantiNova $^{\mathrm{TM}}$ Reverse Transcription Kit (QIAGEN, Germany) according to the manufacturer's recommendations. Flaviviruses NS5 gene fragments were amplified from cDNA employing the oligonucleotide primers shown in Table 2. The amplicons were purified (TransGen, Beijing, China) and then each cDNA was cloned into the pEASYR-T1 Simple Cloning Vector (TransGen, Beijing, China) and transformed into E. coli Trans1-T1 cells. Plasmid DNAs were purified using the EasyPureR Plasmid MiniPrep Kit (TransGen, Beijing, China) according to the manufacturer's instructions. The DNAs were quantified by a NanoDrop-1000 spectrophotometry (Thermo Fisher Scientific, USA). The copy numbers of the DNA were calculated based on the concentration, and 10-fold serial dilutions of this DNA from $10^{9}$ to $10^{0}$ copies per reaction were used as a standard in all heminested reverse transcriptase polymerase chain reactions (hnRT-PCR).

\section{Sensitivity and Specificity of the hnRT-PCR Assay}

The analytical sensitivity of the hnRT-PCR assay was determined by quantification with external standards using serially diluted plasmids $\left(10^{9}\right.$ $10^{0}$ copies $/ \mu \mathrm{L}$ ) containing JEV, DENV-2, WNV, and YFV. For specificity testing, we used cDNA of JEV, DENV-2, YFV, WNV, SINV, and CHIKV. We also used mosquito samples spiked with cDNA of JEV, DENV-2, YFV, and WNV, and the cDNA of mosquito samples was used as control.

\section{Field-caught Mosquitoes}

Mosquito samples were collected at different sites in Guizhou Province between July to August 2018. The collected mosquitoes were frozen and identified quickly and then pooled by species into groups of up 50 individuals. Viral RNAs were extracted and reverse transcribed into cDNA and the hnRT-PCR was performed using designed primers shown in Table 2.

\section{RESULTS}

In order to determine the sensitivity of the hnRTPCR, we examined the lowest detection limits with external standards employing serial dilutions of quantified plasmid DNAs. The lowest detection limits in the first round PCR for JEV, YFV, and WNV were $3 \times 10^{7}, 3 \times 10^{9}$, and $3 \times 10^{7}$ copies $/ \mu$ L. DENV -2 had never been detected. In the second round PCR, there was a substantial increase in sensitivity and the lowest detection limits of JEV, DENV-2, YFV, and WNV were $3 \times 10^{4}, 3 \times 10^{6}, 3 \times 10^{5}$, and $3 \times 10^{4}$ copies $/ \mu \mathrm{L}$, respectively (Figure 1). Therefore, through two rounds of amplification, the sensitivity of hnRT-PCR was increased 10,000 times, which greatly improved the sensitivity and made up for the possibility that conventional PCR could not be detected. For specificity testing, we used cDNA of JEV, DENV-2, YFV, WNV, SINV, and CHIKV. We also used mosquito samples spiked with cDNA of JEV, DENV2, YFV, and WNV (JEV+mosquito cDNA, DENV2+mosquito cDNA, YFV+mosquito cDNA, and $\mathrm{WNV}+$ mosquito cDNA), and cDNA of mosquito samples as control. The results showed that only the target bands appeared in the flavivirus and that no bands appeared in the control, suggesting that the test was $100 \%$ specific for the detection of flavivirus (Figure 2).

Overall, a total of 96 pools of mosquitoes belonging to 4 species of 3 genera were used to examine the sensitivity of the hnRT-PCR assay. Among all mosquitoes collected, $34 \%$ were Culex tritaeniorhynchus, $2.3 \%$ were Culex pipiens pallens, $24.7 \%$ were Anopheles sinensis, and $39 \%$ were Armigeres subalbatus. JEV was detected in 2 mosquito pools out of 96 from Culex tritaeniorhynchus and Armigeres subalbatus. One was identified as type JEV-I isolated from Culex tritaeniorhynchus, and the other was identified as type JEV-III isolated from Armigeres subalbatus.

\section{DISCUSSION}

Flaviviruses are single-stranded RNA viruses with 
TABLE 1. Sequence alignment of oligonucleotide XF-F1, XF-F2, and XF-R with 36 flavivirus NS5 gene conserved regions.

\begin{tabular}{|c|c|c|c|}
\hline \multicolumn{4}{|c|}{ Sequence of oligonucleotide ${ }^{*}+\dagger$} \\
\hline Region & XF-F1 (8916-8938) & XF-F2 (8964-8985) & XF-R (9153-9178) \\
\hline Primer & AACATGATGGGVAARMGWGARAA & AARGGMAGYMGNGCHATHTGGT & GTRTCCCANCCDGCDGTRTCATCNGC \\
\hline Nakayama & AACATGATGGGAAAAAGAGAGAA & AAGGGAAGCAGGGCCATTTGGT & GCTGATGATACCGCCGGGTGGGACAC \\
\hline $\mathrm{SA}(\mathrm{A})$ & $\ldots$ - . - A- AA-A- - G- - & - - A- - A- - CA- G- - C- - T- - - & - - T- - - - T- - C- - C- - G- - - - C- - \\
\hline Beijing-1 & $\ldots-\ldots$ - . - AA- A- - A- & - - A- - A- - CA- G- - C- - T- - - & - - T- - - - T- - C- - T- - G- - - - C- - \\
\hline GP78 & $-\ldots-\ldots$ - - AA- A- - G- & - - A- - A- - CA- G- - C- - C- - - & $--T---$ - C- - C- - C- - G- - - C- - \\
\hline $\mathrm{HVI}$ & $-\ldots$ - . - A- - AA- A- - G- - & - - A- - A- - CA- G- - C- - T- - - & - - T- - - - T- - C- - C- - G- - - - C- - \\
\hline JaGAr01 & $\ldots$ - . . - A- AA- A- - G- & - - A- - A- - CA- G- - C- - T- - - & -- T- - - - T- - C- - C- - G- - - - C- - \\
\hline JaOArS982 & $-\ldots$ - . - A- AA- - G- & - - A- - A- - CA- G- - C- - T- - - & $--T---$ - C- - C- - C- - A- - - - C- - \\
\hline K94P05 & $\ldots$ & - - A- - A- - CA- G- - C- - T- - - & $--C-\ldots-C--C--C-$ G- - - C- - \\
\hline SA-14 & $\ldots-\ldots$ - . - A- AA- A- - G- - & - - A- - A- - CA- G- - C- - T- - - & - - T- - - - T- - C- - C- - G- - - - C- - \\
\hline HawO3663 & $\ldots \ldots$ - . . - - A- GA- A- - G- - & -- A- - A- - TC- C- - A- - A- - - & $--A-\cdots$ C- - A- - C- - A- - - C- - \\
\hline $71 / 02 G Z$ & $-\ldots-\ldots$ - - - A- GA- A- - G- - & -- A- - A- - TC- C- - A- - A- - - & $--A---C--A--C--A---C--$ \\
\hline Nauru Island & $\ldots$ & -- A- - A- - TC- C- - A- - A- - - & -- A- - - C- - A- - C- - A- - - C- - \\
\hline 16681 & $\ldots$ - . - A- AA- A- - G- & $--A--$ C- - CA-A- - C- - A- - - & - - C- - - C- - C- - A- - A- - - C- - \\
\hline New Guinea C & $-\ldots$ - A- AA- A- - G- & $--A--C--C A-A--C--A-\cdots$ & $--C-\cdots$ C- - C- - A- - A- - - C- - \\
\hline PUO-218 & $-\ldots$ - . - AA- A- - G- - & -- A- - C- - CA- A- - C- - A- - - & $--C---C--C-A--A---C--$ \\
\hline $\mathrm{H} 87$ & - . - . - - - C- - GA- A- - G- - & - - A- - C- - TA- G- - T- - A- - - & -- T- - - - C- - A- - C- - T- - - - C- - \\
\hline $80-2$ & $\ldots \ldots$ - . . - . - GA- A- - G- - & -- A- - C- - TA- G- - T- - A- - - & -- T- - - - C- - A- - C- - T- - - - C- - \\
\hline P4 & $-\ldots$ - . - A- AC- T- - G- - & -- G- - A- - CC- A- - A- - C- - - & -- T- - - - C- - A- - A- - C- - - C- - \\
\hline P75-215 & $-\ldots--_{-}-A_{-}$AC- T- - G- & - - A- - A- - CC- G- - A- - T- - - & $--T---$ - C- - A- - A- - T- - - - C- - \\
\hline 11070 & $-\ldots$ - . - - A- - AC- T- - G- - & - - G- - A- - CC- A- - A- - C- - - & -- T- - - - C- - A- - A- - C- - - - C- - \\
\hline serum & $\ldots$. . . . - A- GA- A- - G- - & - - G- - A- - CA- A- - C- - T- - - & -- T- - - - C- - A- - T- - C- - - - C- - \\
\hline FtC-3699 & $-\ldots-\ldots$ - . - - GA- A- - G- - & - - G- - A- - CA-A- - C- - T- - - & -- T- - - - C- - A- - T- - C- - - - C- - \\
\hline $385-99$ & - - - - - A- - GA- A- - G- - & - - G- - A- - CA- A- - C- - T- - - & 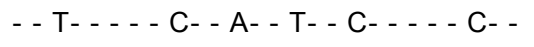 \\
\hline ARC13-12 & - - - . - - - A- - GA- A- - G- - & - - G- - A- - CA- A- - C- - T- - - & - - T- - - - C- - A- - T- - C- - - - C- - \\
\hline Chin-01 & $-\ldots$ - - - - G- GA- A- - A- - & -- G- - A- - CA-A- - C- - A- - - & -- T- - - - C- - A- - T- - C- - - C- - \\
\hline 33-G8 & $-\ldots-\ldots$ - - - - GA- A- - G- - & $--A--C--C A-A--C--C---$ & -- T- - - - T- - C- - A- - C- - - - C- - \\
\hline HNY1999 & $\ldots \ldots$ - . . - . - GA- A- - G- - & - - G- - A- - CA- A- - C- - T- - - & -- T- - - - C- - A- - T- - C- - - - C- - \\
\hline 17D vaccine & - . - . - - G- AA- A- - G- - & -- G- - A- - CC- T- - C- - A- - - & - - G- - - - C- - C- - T- - A- - - C- - \\
\hline $\begin{array}{l}\text { French } \\
\text { viscerotropic }\end{array}$ & - . . - . - - G- - AA- A- - G- - & - - G- - A- - CC- T- - C- - A- - - & -- G- - - - C- - C- - T- - A- - - - C- - \\
\hline $17 \mathrm{D}$ vaccine & $\ldots$ & - - G- - A- - CC- T- - C- - A- - - & -- G- - - - C- - C- - T- - A- - - - C- - \\
\hline 17DD & - - - . - - - G- - AA- A- - G- - & - - G- - A- - CC- T- - C- - A- - - & - - G- - - - C- - C- - T- - A- - - - C- - \\
\hline 17D-213 & - - - - - - G- AA- A- - G- - & - - G- - A- - CC- T- - C- - A- - - & - - G- - - - C- - C- - T- - A- - - - C- - \\
\hline $85-82 \mathrm{H}$ & - . . - . - - G- AA- A- - G- - & -- A- - A- - CC- T- - C- - C- - - & - - G- - - - T- - C- - T- - G- - - - C- - \\
\hline $\begin{array}{l}\text { French } \\
\text { viscerotropic }\end{array}$ & - . - . - - G- AA- A- - G- - & -- G- - A- - CC- T- - C- - A- - - & -- G- - - - C- - C- - T- - A- - - - C- - \\
\hline Trinidad 79A & $\ldots$ & - - G- - A- - CC- T- - C- - A- - - & -- G- - - - C- - C- - T- - A- - - - C- - \\
\hline $\begin{array}{l}\text { French } \\
\text { viscerotropic } \\
\text { virus }\end{array}$ & $-\ldots-\ldots$ & - - G- - A- - CC- T- - C- - A- - - & - - G- - - - C- - C- - T- - A- - - - C- - \\
\hline
\end{tabular}

${ }^{*} \mathrm{~N}=\mathrm{A}+\mathrm{G}+\mathrm{C}+\mathrm{T}, \mathrm{V}=\mathrm{G}+\mathrm{A}+\mathrm{C}, \mathrm{R}=\mathrm{A}+\mathrm{G}, \mathrm{M}=\mathrm{A}+\mathrm{C}, \mathrm{W}=\mathrm{A}+\mathrm{T}, \mathrm{Y}=\mathrm{C}+\mathrm{T}, \mathrm{H}=\mathrm{A}+\mathrm{T}+\mathrm{C}, \mathrm{D}=\mathrm{G}+\mathrm{A}+\mathrm{T}$

${ }^{\dagger}$ DNA sequence were obtained from the GenBank databases. Accession numbers are as follows: Nakayama, EF571853.1; $S A(A)$, D90195.1; Beijing-1, L48961.1; GP78, AF075723.1; HVI, AF098735.1; JaGAr01, AF069076.1; JaOArS982, M18370.1; K94P05, AF045551.2; SA-14, M55506.1; HawO3663, DQ672564.1; 71/02GZ, EF025110.1; Nauru Island, U88535.1; 16681, U87411.1; New Guinea C, AF038403.1; PUO-218, AF038402.1; H87, M93130.1; 80-2, AF317645.1; P4, AY648301.1; P75-215, EF457906.1; 11070, M14931.2; serum, AY646354.1; FtC-3699, KR868734.1; 385-99, AY842931.3; ARC13-12, KM012188.1; Chin-01, AY490240.2; 33-G8, M12294.2; HNY1999, AF202541.1; 17D vaccine, X03700.1; French viscerotropic, U21056.1; 17D vaccine, NC_002031.1; 17DD, U17066.1; 17D-213, U17067.1; 85-82H, U54798.1; French viscerotropic, U21055.1; Trinidad 79A, AF094612.1; French viscerotropic virus, U21056.1. 
TABLE 2. Nucleotide sequences and positions of primers used in the heminested RT-PCR (hnRT-PCR) assay for detection of mosquito-borne flavivirus.

\begin{tabular}{|c|c|c|c|c|c|}
\hline & Primer & Sequence $\left(5^{\prime}-3^{\prime}\right)^{*}$ & Nucleotide position & Annealing temperature $\left({ }^{\circ} \mathrm{C}\right)$ & Length (bp) \\
\hline \multirow{2}{*}{$1^{\text {st }}$ round PCR } & $\mathrm{XF}-\mathrm{F} 1$ & AACATGATGGGVAARMGWGARAA & $8916-8938$ & \multirow{2}{*}{52} & \multirow{2}{*}{263} \\
\hline & XF-R & GTRTCCCANCCDGCDGTRTCATCNGC & $9153-9178$ & & \\
\hline \multirow{2}{*}{$2^{\text {nd }}$ round $P C R$} & $\mathrm{XF}-\mathrm{F} 2$ & AARGGMAGYMGNGCHATHTGGT & 8964-8985 & \multirow{2}{*}{54} & \multirow{2}{*}{215} \\
\hline & $X F-R$ & GTRTCCCANCCDGCDGTRTCATCNGC & $9153-9178$ & & \\
\hline
\end{tabular}

${ }^{*} \mathrm{~N}=\mathrm{A}+\mathrm{G}+\mathrm{C}+\mathrm{T}, \mathrm{V}=\mathrm{G}+\mathrm{A}+\mathrm{C}, \mathrm{R}=\mathrm{A}+\mathrm{G}, \mathrm{M}=\mathrm{A}+\mathrm{C}, \mathrm{W}=\mathrm{A}+\mathrm{T}, \mathrm{Y}=\mathrm{C}+\mathrm{T}, \mathrm{H}=\mathrm{A}+\mathrm{T}+\mathrm{C}, \mathrm{D}=\mathrm{G}+\mathrm{A}+\mathrm{T}$.

A1

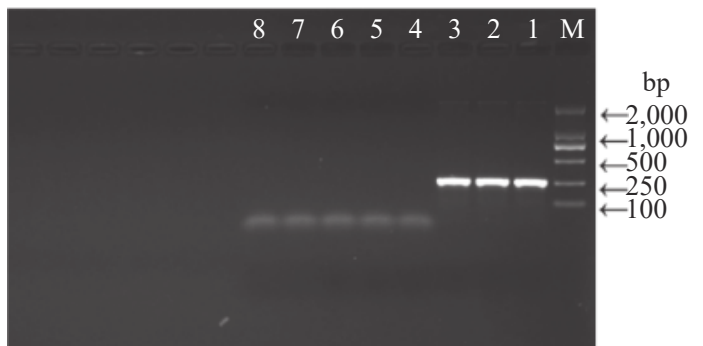

A2

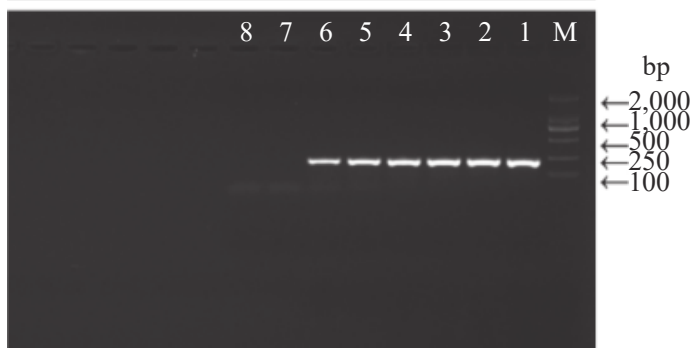

$\mathrm{C} 1$

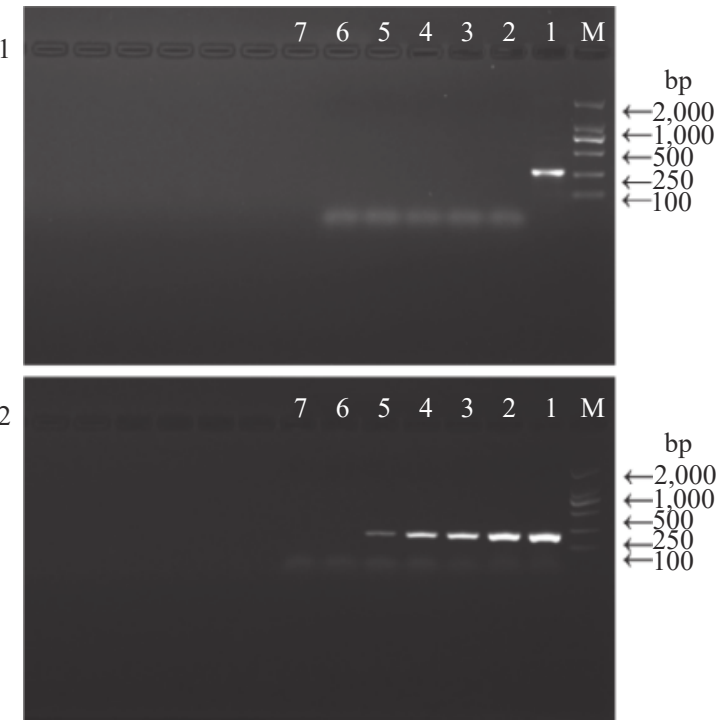

B1

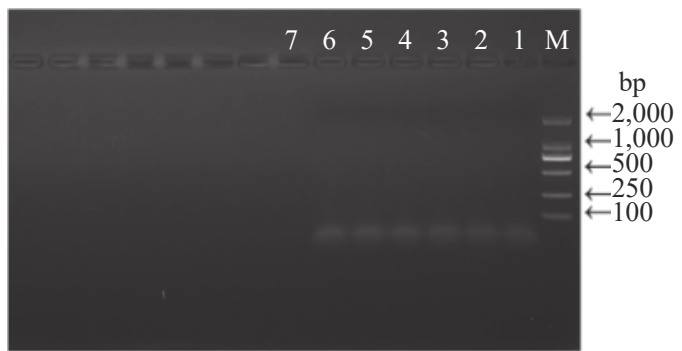

B2

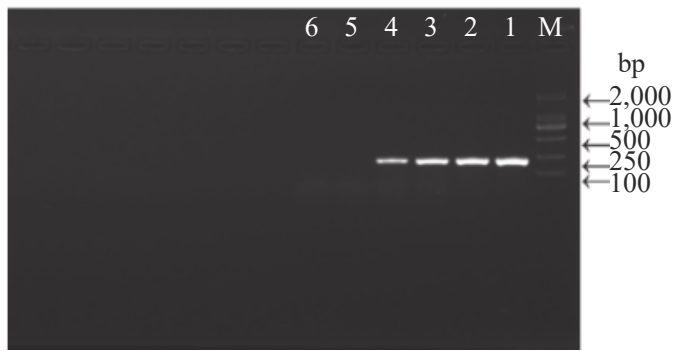

$\mathrm{D}$

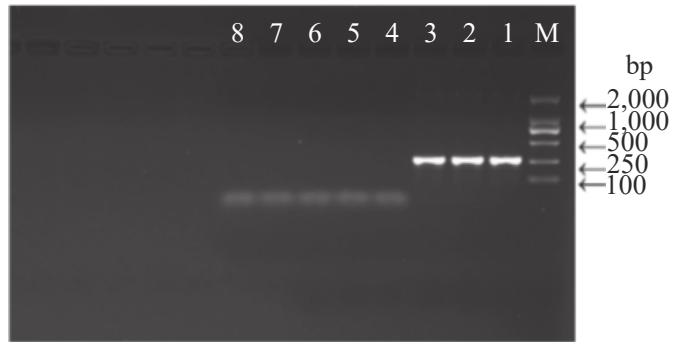

D2

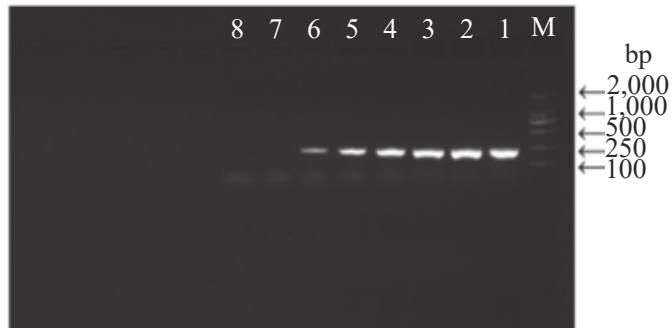

FIGURE 1. Sensitivity of heminested RT-PCR (hnRT-PCR) for detection of mosquito-borne flavivirus.

$A, B, C$, and D show the lowest limit for JEV, DENV-2, YFV, and WNV, respectively; 1 and 2 denote the lowest limit for the first and second round PCR, respectively. The numbering $1-8$ refers to $10^{9}-10^{3}$ copies/ $\mathrm{L}$ and negative control, respectively. M: DL2000 DNA markerr.

various species and are widely distributed worldwide. Most flaviviruses are pathogens causing natural infectious diseases, which can lead to fever, hemorrhage, and encephalitis with a high mortality rate (9). In this study, four flaviviruses were screened based on the epidemic situation, pathogenicity, and risk of disease, including JEV, DENV-2, WNV, and YFV. Among them, JEV and DENV-2 are the 


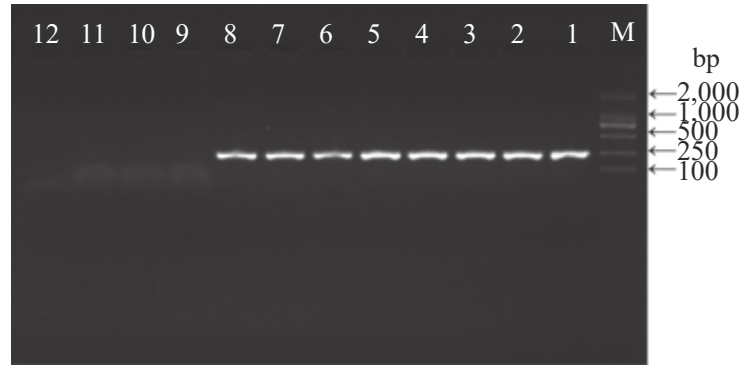

FIGURE 2. Specificity of heminested RT-PCR (hnRT$\mathrm{PCR}$ ) for detection of mosquito-borne flavivirus.

The numbering 1-12 refers to JEV, DENV-2, YFV, WNV, JEV + mosquito cDNA, DENV-2 + mosquito cDNA, YFV + mosquito cDNA, WNV + mosquito cDNA, mosquito cDNA, SINV, CHIKV, and negative control, respectively. M: DL2000 DNA marker.

common mosquito-borne viruses, and associated outbreaks occur almost every year in China. Therefore, the rapid and accurate high-throughput detection method for flavivirus is of great significance for the prevention and control of mosquito-borne diseases.

The traditional methods for detecting mosquitoborne viruses are isolation and culturing method, but virus culturing is relatively time-consuming and cannot be used for early diagnosis of disease. Serological detection is a classical virus detection method, which is mainly used to detect specific antibodies or antigens in specimens, and it is indispensable in the diagnosis and identification of virus infection. But serological methods lack of specificity and are prone to crossreactions between viruses. Traditional species-specific RT-PCR and real-time RT-PCR based on Taq-Man Probe are rapid and sensitive, but only detect single pathogens.

Wu et al. (10) established a method for screening samples by multiplex real-time RT-PCR and the lower limit of detection of this method was 11 copies $/ \mu \mathrm{L}$. However, compared with singleplex real-time PCR, multiplex real-time PCR requires multiple pairs of primers and probes in a single reaction tube and, therefore, will have to account for competition between reactants resulting in significantly increased difficulty of primer design. Gao et al. (11) used the established RT-PCR method to detect JEV, and the lower limit of detection was $10 \mathrm{pg} / \mu \mathrm{l}$ but could only detect a single flavivirus.

In this study, a new hnRT-PCR method for the simultaneous detection of various flaviviruses was established. The performance of the new hnRT-PCR method was evaluated including specificity and sensitivity. In the specificity testing, only the target bands appeared in the flavivirus, and no bands appeared in control, which indicated high specificity in detecting mosquito-borne flavivirus. In the sensitivity testing, the lowest detection limits of JEV, DENV-2, $\mathrm{YFV}$, and WNV were $3 \times 10^{4}, 3 \times 10^{6}, 3 \times 10^{5}$, and $3 \times 10^{4}$ copies $/ \mu \mathrm{L}$, respectively. When testing fieldcaught mosquito samples, 2 strains of JEV were detected by the method established in this study. Other flaviviruses had not been detected, which may be related to the species and quantity of mosquitoes collected, and the epidemic situation of the virus. However, considering that the variety and high genetic diversity of flavivirus, whether the hnRT-PCR method established in this study had a good detection effect for all flavivirus viruses needs to be further validated by other flavivirus viruses. In summary, the hnRT-PCR established in this study possessed both high specificity and sensitivity and could simultaneously be used for detection of multiple flaviviruses. This can be effectively used to detect the pathogen of flavivirus and provide technical support for early and rapid diagnosis of mosquito-borne flavivirus.

Acknowledgment: Guizhou Province CDC.

Funding: the National Important Scientific \& Technology Project (2018ZX10101002-002) and China Prosperity Strategic Programme Fund (SPF) 2015-16 (15LCI1).

doi: $10.46234 / \mathrm{ccd}$ cw2 2021.001

\# Corresponding authors: Zhong Zhang, nasonia@163.com; Qiyong Liu, liuqiyong@icdc.cn.

1 National Institute for Communicable Disease Control and
Prevention, Chinese Center for Disease Control and Prevention,
Beijing, China; ${ }^{2}$ School of Basic Medical Sciences, Shandong First
Medical University, Taian, Shandong, China; ${ }^{3}$ Guizhou Province
Center for Disease Control and Prevention, Guiyang, Guizhou, China;
${ }^{4}$ School of Life Sciences, Shandong First Medical University, Taian,
Shandong, China; ${ }^{5}$ Collaborative Innovation Center for the Origin
and Control of Emerging Infectious Diseases, Taian, Shandong, China.

Submitted: September 07, 2020; Accepted: December 28, 2020

\section{REFERENCES}

1. Weaver SC, Reisen WK. Present and future arboviral threats. Antiviral Res 2010;85(2):328 - 45. http://dx.doi.org/10.1016/j.antiviral.2009. 10.008.

2. Erlanger TE, Weiss S, Keiser J, Utzinger J, Wiedenmayer K. Past, present, and future of Japanese encephalitis. Emerg Infect Dis 2009; 15(1):1 - 7. http://dx.doi.org/10.3201/eid1501.080311.

3. Gould E, Pettersson J, Higgs S, Charrel R, de Lamballerie X. Emerging arboviruses: why today? One Health 2017;4:1 - 13. http://dx.doi.org/ 10.1016/j.onehlt.2017.06.001.

4. Tang KF, Ooi EE. Diagnosis of dengue: an update. Expert Rev AntiInfect Ther 2012;10(8):895 - 907. http://dx.doi.org/10.1586/eri.12.76.

5. Asnis DS, Conetta R, Teixeira AA, Waldman G, Sampson BA. The west Nile virus outbreak of 1999 in New York: the flushing hospital 
experience. Clin Infect Dis 2000;30(3):413 - 8. http://dx.doi.org/ $10.1086 / 313737$.

6. Franca RFO, Neves MHL, Ayres CFJ, Melo-Neto OP, Filho SPB. First international workshop on Zika virus held by Oswaldo Cruz foundation FIOCRUZ in Northeast Brazil March 2016-a meeting report. PLoS Negl Trop Dis 2016;10(6):e0004760. http://dx.doi.org/10.1371/ journal.pntd.0004760

7. Jarman RG, Nisalak A, Anderson KB, Klungthong C, Thaisomboonsuk $\mathrm{B}$, Kaneechit W, et al. Factors influencing dengue virus isolation by C6/36 cell culture and mosquito inoculation of nested PCR-positive clinical samples. Am J Trop Med Hyg 2011;84(2):218 - 23. http://dx.doi.org/10.4269/ajtmh.2011.09-0798.

8. Lu Z, Lu XJ, Fu SH, Zhang S, Li ZX, Yao XH, et al. Tahyna virus and human infection, China. Emerg Infect Dis 2009;15(2):306-9. http://dx.doi.org/10.3201/eid1502.080722.
9. Yang CF, Chen CF, Su CL, Teng HJ, Lu LC, Lin C, et al. Screening of mosquitoes using SYBR Green I-based real-time RT-PCR with groupspecific primers for detection of Flaviviruses and Alphaviruses in Taiwan. J Virol Methods 2010;168(1 - 2):147 - 51. http://dx.doi.org/ 10.1016/j.jviromet.2010.05.006.

10. Wu WH, Wang J, Yu N, Yan JY, Zhuo ZH, Chen MY, et al. Development of multiplex real-time reverse-transcriptase polymerase chain reaction assay for simultaneous detection of Zika, dengue, Yellow fever, and chikungunya viruses in a single tube. J Med Virol 2018;90(11):1681 - 86. http://dx.doi.org/10.1002/jmv.25253.

11. Gao ZQ, Yue BF, He ZM. Development and application of RT-PCR assay of Japanese encephalitis virus. Chin J Pharm Anal 2010;30(10): 1928-31. http://d.wanfangdata.com.cn/periodical/ChlQZXJpb2RpY2 FsQ0hJTmV3UzIwMjAxMjI4Eg95d2Z4enoyMDEwMTAwMjgaCDF 6Y2tsem9t. (In Chinese). 